\title{
Characterisation of the optical channel GEO to ground: using five years of data from Alphasat TDP1 and T-AOGS for investigation of different conditions
}

K. Saucke, J. Woicke, R. Mahn, T. Marynowski, P. Martin Pimentel, et al.

K. Saucke, J. Woicke, R. Mahn, T. Marynowski, P. Martin Pimentel, F. Heine, J. Surof, A. Reeves, R. Mata-Calvo, "Characterisation of the optical channel GEO to ground: using five years of data from Alphasat TDP1 and T-AOGS for investigation of different conditions," Proc. SPIE 11852, International Conference on Space Optics - ICSO 2020, 118525W (11 June 2021); doi: $10.1117 / 12.2600018$

SPIE Event: International Conference on Space Optics - ICSO 2021, 2021, Online Only 


\section{International Conference on Space Optics-ICSO 2020}

Virtual Conference

30 March-2 April 2021

Edited by Bruno Cugny, Zoran Sodnik, and Nikos Karafolas
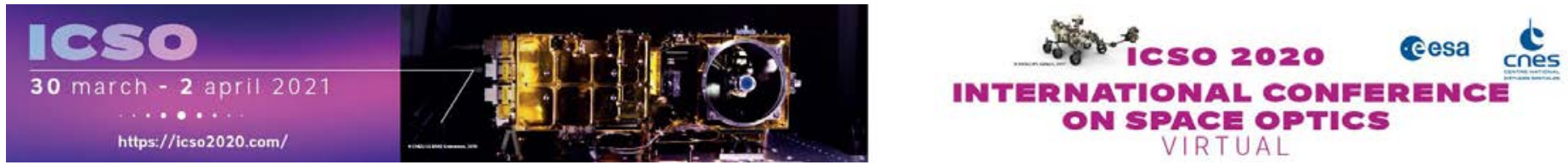

\section{Characterisation of the optical channel GEO to ground: using five years of data from Alphasat TDP1 and T-AOGS for investigation of different conditions}

\section{Cesa issopoesealins ecnes}




\title{
Characterisation of the optical channel GEO to ground: using five years of data from Alphasat TDP1 and T-AOGS for investigation of different conditions
}

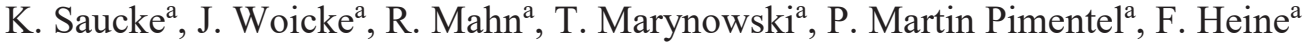 \\ J. Surof ${ }^{\mathrm{b}}$, A. Reeves ${ }^{\mathrm{b}}$, R. Mata-Calvo ${ }^{\mathrm{b}}$ \\ ${ }^{a}$ Tesat Spacecom, Backnang, Germany \\ ${ }^{b}$ Institute of Communications und Navigation, DLR, Wessling, Germany
}

\begin{abstract}
An important step towards reliable optical communication between ground and space is the characterization and understanding of atmospheric conditions - even when being at one place and performing links into one direction, but at different times. Within this paper the analysis of five years of optical links from a laser terminal on a geostationary satellite (TDP1-LCT on Alphasat) to ground (T-AOGS at Tenerife) is shown. The data, in total $40.2 \mathrm{~h}$ of reliable measurements extracted from 516 performed links, are spread over several times of the year and almost all times of day. For one of the main parameters describing the atmosphere (the Fried Parameter r0) a clear characteristic over the day is validated. For the up-link the influence of different transmitted beam divergence angles on the link budget in terms of mean power and fade statistic is shown. A short outlook on upcoming changes within the TDP-1 software and the TAOGS functionality is given.
\end{abstract}

Keywords: optical satellite links, atmospheric channel, Fried Parameter r0, fading and scintillation, link budget, beam divergence

\section{INTRODUCTION}

Since September 2015 the Transportable Adaptive Optical Ground Station (T-AOGS 2, 3, 4), co-located with the European Space Agency (ESA) Optical Ground Station on Tenerife, has been operating and performing links with the laser terminal (LCT) on the geostationary spacecraft $(\mathrm{S} / \mathrm{C})$ Alphasat ${ }^{1}$. Since then more than 630 Satellite to Ground Links (SGLs) were carried out under different conditions ${ }^{5}$, e.g. day/night, but also with different transmitter apertures and thus divergence angles of the T-AOGS. With the TDP1-LCT on Alphasat in the geostationary orbit at $25^{\circ}$ East, inclination of $\sim 4^{\circ}$ and the T-AOGS being located at (in WGS84): Longitude: $-16.51 \mathrm{deg}$, Latitude: $+28.30 \mathrm{deg}$ and Altitude: $2.45 \mathrm{~km}$, the SGLs probe the atmosphere at an elevation of $34^{\circ}+/-4^{\circ}\left(\mathrm{El}=90^{\circ}\right.$ would be zenith) and to a south east direction (Az $=118^{\circ}$, clockwise from north).

The TDP1-LCT is built as a communication terminal with coherent homodyne optical communication using $1064 \mathrm{~nm}$ and binary phase shift keying (BPSK) with a clock rate of $2.8125 \mathrm{Gbps}$ and a standard user data rate of $1.8 \mathrm{Gbps}$, or alternatively $600 \mathrm{Mbps}$, which can be sent to ground using the TDP1-Ka-band antenna ${ }^{1}$. Additionally, the LCT can also be used as a measurement device for the received optical light while performing tracking or communication links. Besides other parameter, the received signal is measured at $25 \mathrm{kHz}$ and transferred, instead of user data, in full data rate to ground via the TDP1-Ka-band system. The detector used for measurement is the tracking and communication detector. Its electronic signal has two regimes: either heterodyne or homodyne. For optical communication only the homodyne signals are relevant, but for characterization the optical channel both, time spans with heterodyne or homodyne signal can be taken, but need to be calibrated separately.

In the course of the data analysis the calibration of the receiver signal in $\mathrm{V}$ to received optical power in $\mathrm{W}$ has been revised. While the terminal is designed for a received optical power of $-44 \mathrm{dBm}$ in the $135 \mathrm{~mm}$ aperture of the Coarse Pointing Assembly (CPA), which equals a received power of $40 \mathrm{nW}$ or a power area density of $0.28 \mathrm{nW} / \mathrm{cm}^{2}$, the dynamic range is $19 \mathrm{~dB}$, from $-54 \mathrm{dBm}$ to $-35 \mathrm{dBm}$. When measuring in the heterodyne regime only, a dynamical range of $22 \mathrm{~dB}$ can be reached. These values are summarized in Table 1 . The absolute calibration and thus measurement 
accuracy is $<20 \%$. This is due to the fact that on ground calibrations and measurements were done in the spirit of proving requirements, but not calibrating a measurement device.

Table 1. Calibrated measurement range of the TDP1-LCT, being in homodyne or heterodyne mode

\begin{tabular}{|c|l|l|l|l|}
\hline Link type & \multicolumn{2}{|c|}{ Minimum received power } & \multicolumn{2}{c|}{ Maximum received power } \\
\hline & in $135 \mathrm{~mm}$ aperture & & in $135 \mathrm{~mm}$ aperture & \\
\hline homodyne & $3.7 \mathrm{nW}=-54 \mathrm{dBm}$ & $0.026 \mathrm{nW} / \mathrm{cm} 2$ & $350 \mathrm{nW}=-35 \mathrm{dBm}$ & $2.45 \mathrm{nW} / \mathrm{cm} 2$ \\
\hline heterodyne & $3.7 \mathrm{nW}=-54 \mathrm{dBm}$ & $0.026 \mathrm{nW} / \mathrm{cm} 2$ & $700 \mathrm{nW}=-32 \mathrm{dBm}$ & $4.89 \mathrm{nW} / \mathrm{cm} 2$ \\
\hline
\end{tabular}

The T-AOGS, which serves as a measurement device on the ground has an InGaAs pin photodiode as direct detector, thus the signal in $\mathrm{V}$ is proportional to the power of light received. No further calibration to optical power has yet been performed. The data are taken at a rate of $5 \mathrm{kHz}$. Besides the received power, the T-AOGS, as equipped with an adaptive optics system in the receive path which also measures the Fried-Parameter, $r_{0}$ (see Ref. 4, but also section 5.4.1). The TAOGS can be used in different configurations (see also Ref. 2, 3, 4). The transmitter aperture diameter can be changed and the following three options are used: $35 \mathrm{~mm}, 48 \mathrm{~mm}$ and $95 \mathrm{~mm}$ TX aperture $\left(2 * w_{0} * 1.12\right)$, which leads to a $1 / \mathrm{e}^{2}$ beam divergence radius of $26 \mu \mathrm{rad}, 19 \mu \mathrm{rad}$ and $9.6 \mu \mathrm{rad}$ (considering also $\sim 20 \%$ for truncation). One further detail should be mentioned: the T-AOGS always uses a $270 \mathrm{~mm}$ aperture, the CPA270, for receiving and tracking light. The transmit path, which is already separated from the receive path at an early stage, either sends through the same CPA270, or, most often, through a second coarse pointing assembly, the CPA100. The CPA100 follows the tracking path of the CPA270, but a) the alignment of the receive and the transmit path is not stable at all time, and must be aligned during links and b) the optical path of received and transmit light is not the same.

\section{AUTOMATED PROCESSING OF DATA}

There have been five years worth of data recorded. This represents 1541 commanded SGLs in total with automated telemetry recording at TDP1-LCT, and of this 630 links successfully executed with the T-AOGS and the corresponding telemetry recording: this amount of data cannot be analysed manually and link per link. For the LCTs in orbit ${ }^{8}$ TESAT has set up an automated analysis too ${ }^{6}$, the long term and performance analysis tool LTPA. This is also used for the TDP1-LCT, but only based on $1 \mathrm{~Hz}$ telemetry data. To analyse SGLs and the optical channel through atmosphere, the analysis of the high data rate telemetry $(25 \mathrm{kHz}$ and $5 \mathrm{kHz})$ is necessary. An automated way to process and analyse the data is set up. This is done as part of a joined project called ATLANTIS and we call the main part of the data processing script the ATLANTIS tool. For a correct understanding and interpretation of the results the way of extraction and processing is useful and thus described in the following section.

\subsection{Pre-Processing of data: identify suitable times and extract data.}

As a first step all times where the TDP1-LCT receives light are identified. In order to include also time-spans with deep fades, i.e. light gone for a short time, the script also keeps time-spans where the space segment had seen sufficient light, i.e. being in tracking, but afterwards loses the light - for shorter periods (fades), but also for longer periods.

Once the list of suitable time-spans of the relevant data is identified, the relevant telemetries are extracted out of the available $25 \mathrm{kHz}$ (TDP1) and $5 \mathrm{kHz}$ (T-AOGS) data.

There are two drawbacks of this method: a) if the link budget is very good, and the space segment has already begun tracking while the ground segment is still, semi-manually, correcting it's pointing, the data is treated as valid, even though it still contains part of the alignment phase. b) power drops seen at the space segment are not only due to the atmosphere: it was a common operational scenario, that the T-AOGS operator switched off the ground OPA for $\sim 1 \mathrm{~s}$, during several special tests, e.g. re-starting spatial and frequency acquisition. These times are currently part of the prepossessed data, though they are removed in the final processing via an ignore list and dedicated filtering.

In addition to the pure data pre-processing, special information like the used T-AOGS divergence angle or transmitting path (CPA100 or CPA270) are included in the file information. 


\subsection{Processing the data within ATLANTIS tool}

In its first step the ATLANTIS tool generates what we call CHUNKs: each of the 'pre-processed-time-spans' are analysed whether the TPD1-LCT signal is homodyne, heterodyne or unclear and the data are split into CHUNKs of consecutive homodyne signal or consecutive heterodyne signal. The unclear times, which are mainly times where the frequency (re-)acquisition takes place, are ignored because a clear calibration from signal in $\mathrm{V}$ to optical power in $\mathrm{W}$ cannot be given. The TDP1-LCT detector signal is converted to received optical light, and the data from the T-AOGS are added to the data set of each CHUNK, thus the CHUNKs contain the following data: time, $25 \mathrm{kHz}$ space segment received light and $5 \mathrm{kHz}$ ground segment received signal. Each CHUNK is assigned a unique number.

In the following, each CHUNK is evaluated and characteristic information extracted. These are, besides others: mean power, number of fades (for $-3 \mathrm{~dB},-6 \mathrm{~dB},-10 \mathrm{~dB})$, mean duration of fades $(-3 \mathrm{~dB},-6 \mathrm{~dB},-10 \mathrm{~dB})$, both: at TDP1 and at T-AOGS. In order to make the fade analysis robust against electronic noise, the power signal is low pass filtered; and to be robust against slow power variations during a CHUNK, the $-3 \mathrm{~dB},-6 \mathrm{~dB}$, and $-10 \mathrm{~dB}$ is taken relative to a 3 sec local mean values (see also section 4.2). All of this information, together with evaluated T-AOGS data and used setting (e.g. sending aperture) is listed per CHUNK. In addition to a table gathering the information, each CHUNK is visualized with a set of plots: time-line of power, distribution of power, CDF of fade duration etc.

\subsection{Filtering options and final processing within ATLANTIS tool}

Once all CHUNKs are generated, analysed individually and a table with the main information and characteristic (mean) values are generated, the data can be filtered by all table information. In addition, a CHUNK-ignore list can be used. In a first step this ignore list and filter settings are used to clean up the data from all CHUNKs containing obviously incorrect data. e.g. T-AOGS OPA switched off for $2 \mathrm{~s}$ can be seen either in the time-line, but also in a strongly increased mean 'fade' duration.

As the pre-processing is not yet optimized, we lose about $5 \mathrm{~h}$ at this filtering step, i.e. $10 \%$ of the data. But the remaining data are still sufficient for the analysis on 'big-data'.

\section{DATA BEING AVAILABLE}

Table 2. Overview of links with data available and the resulting data used for analysis

\begin{tabular}{|c|c|c|c|c|c|}
\hline $\begin{array}{c}\text { T-AOGS TX } \\
\text { aperture, 1/e2 } \\
\text { divergence angle }\end{array}$ & $\begin{array}{c}\text { Number of } \\
\text { links } \\
\text { performed }\end{array}$ & $\begin{array}{c}\text { Number of } \\
\text { data sets } \\
\text { after pre- } \\
\text { processing }\end{array}$ & $\begin{array}{c}\text { Number } \\
\text { of } \\
\text { CHUNKs }\end{array}$ & $\begin{array}{c}\text { Mean } \\
\text { CHUNK } \\
\text { duration }\end{array}$ & $\begin{array}{c}\text { Total } \\
\text { amount of } \\
\text { good data }\end{array}$ \\
\hline Sum & 516 & 1724 & 2481 & $58 \mathrm{~s}$ & $40.2 \mathrm{~h}$ \\
\hline $35 \mathrm{~mm}, 26 \mu \mathrm{rad}$ & 352 & 1126 & 1728 & $60 \mathrm{~s}$ & $28.9 \mathrm{~h}$ \\
\hline $48 \mathrm{~mm}, 19 \mu \mathrm{rad}$ & 153 & 559 & 715 & $53 \mathrm{~s}$ & $10.7 \mathrm{~h}$ \\
\hline $95 \mathrm{~mm}, 9.6 \mu \mathrm{rad}$ & 11 & 39 & 38 & $51 \mathrm{~s}$ & $0.5 \mathrm{~h}$ \\
\hline
\end{tabular}

Table 2 shows the amount of data available. Starting out with 516 links performed with the T-AOGS and TDP1 having the $25 \mathrm{kHz}$ telemetry channel activated (i.e. the user data dump is deactivated), 352 of these links were performed with the T-AOGS TX aperture of $35 \mathrm{~mm}, 153$ with $48 \mathrm{~mm}$. The $95 \mathrm{~mm}$ aperture is, with 11 links, not used very often: with the given way of tracking and pointing and especially potential TX/RX misalignment, a robust link was difficult to achieve with a narrow beam of $9.6 \mu \mathrm{rad} 1 / \mathrm{e}^{\wedge 2}$ radius. Thus, $35 \mathrm{~mm}$ and $48 \mathrm{~mm}$ were used most the time as the 'workhorse' mode of operation. A performed link has a typical duration of $20 \mathrm{~min}$, from which in average 10 min tracking (or communication) is achieved - thus $\sim 86 \mathrm{~h}$ of data can be expected. The pre-processing stage (section 2.1) generates 1724 single files with a total time duration of $80.4 \mathrm{~h}$. During the data processing in the ATLANTIS tool (section 2.2) 2481 CHUNKs are generated with a total length of $45 \mathrm{~h}$. The total amount of data rejected, i.e. time spans which are neither poor homodyne nor pure heterodyne, and thus a calibration of the receiver signal to optical power is not reliable, is $35 \mathrm{~h}$. 
At first glance, this seems to be a large fraction of the available data, however the aim of the SGLs is not only to measure the optical channel, but also e.g. to test the limits of up-link communication in unstable link conditions, the amount of rejected data only shows the different experimental usage of the SGLs.

The last columns within Table 2. shows the remaining amount of data in hours finally used in the 'big-data analysis' as total and separated to the different T-AOGS TX apertures used. In the following, we call this 'good data'.

Figure 1. shows the distribution of the extracted good data over the year and over the day. The data are taken during several dedicated campaigns, thus the data are not equally distributed over the year (Figure 1. left), but all seasons of the year are addressed. In Figure 1, right it can be seen that the measurements do not sample all times of the day equally.. Most data was recorded in the late evening and early night, very little data was taken during lunch breaks. It can also be seen that the data for the $95 \mathrm{~mm}$ T-AOGS TX aperture were only taken very late in the night, and one trial early in the morning. Both at times with, in average, excellent conditions and thus not representative of the full day. This should be remembered when interpreting the results.
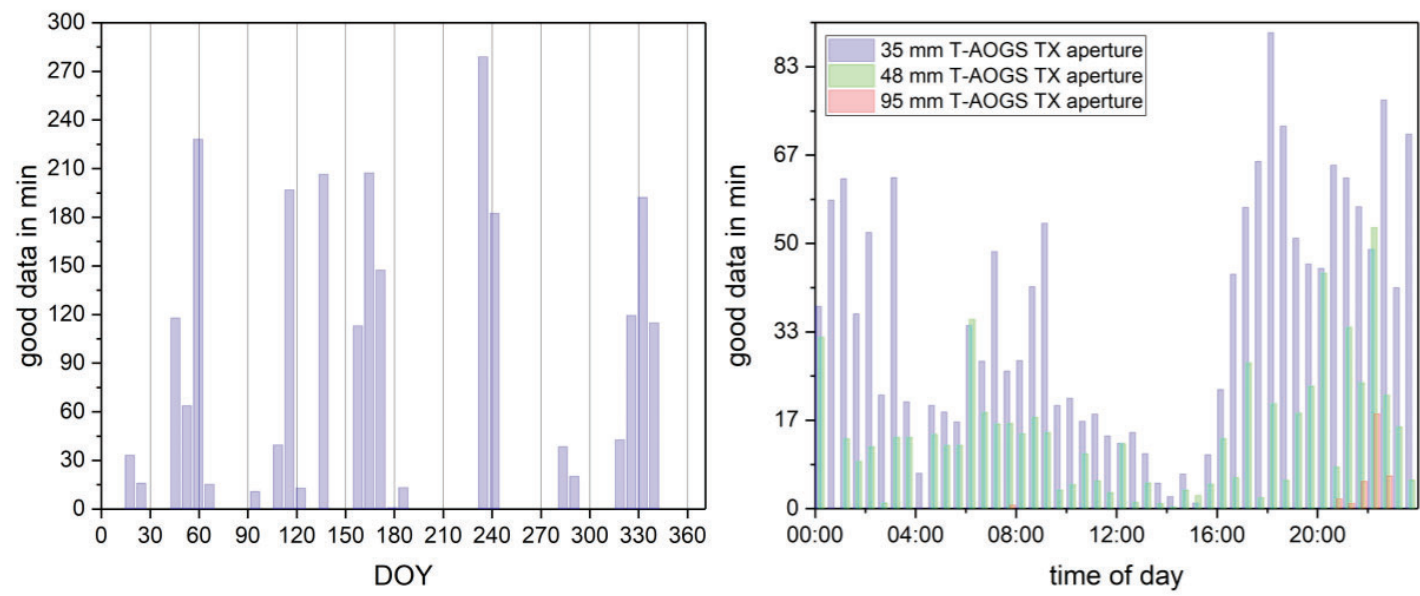

Figure 1. left: total amount of good data as spread over the year in bins of 7 days. The $\mathrm{x}$-axis ticks are monthly. right: total amount of good data, separated to T-AOGS TX aperture as spread over time of day in bins of $30 \mathrm{~min}$.

\section{SINGLE EXAMPLES}

Within the following chapter single CHUNKs are shown as examples: a typical good time-series but also a less good time-series. The later is also investigated regarding the root-cause of the observed behaviour. Both are part of the reliable CHUNKs within the data pool.

\subsection{Link: 2018-04-26, T0 = 00:50, T-AOGS aperture $=35$ mm, CHUNK\#2294}

The single CHUNK shown and described here is representative of a well behaved dataset regarding the optical power received at the space terminal TDP1-LCT. The T-AOGS performing the link using the $35 \mathrm{~mm}$ sending aperture via the CPA100. The link itself started at 00:50:00 on 2018-04-26. Data recording for this CHUNK began at a time of 01:05:48, which is represented as $\mathrm{t}=0$ in Figure 2 .

This single CHUNK has a mean optical power received within the $135 \mathrm{~mm}$ aperture of the LCT in space of $54.7 \mathrm{nW}(-$ $42.6 \mathrm{dBm}$, i.e. $0.38 \mathrm{nW} / \mathrm{cm}^{2}$ ). The fades statistic is shown in Table 3. 

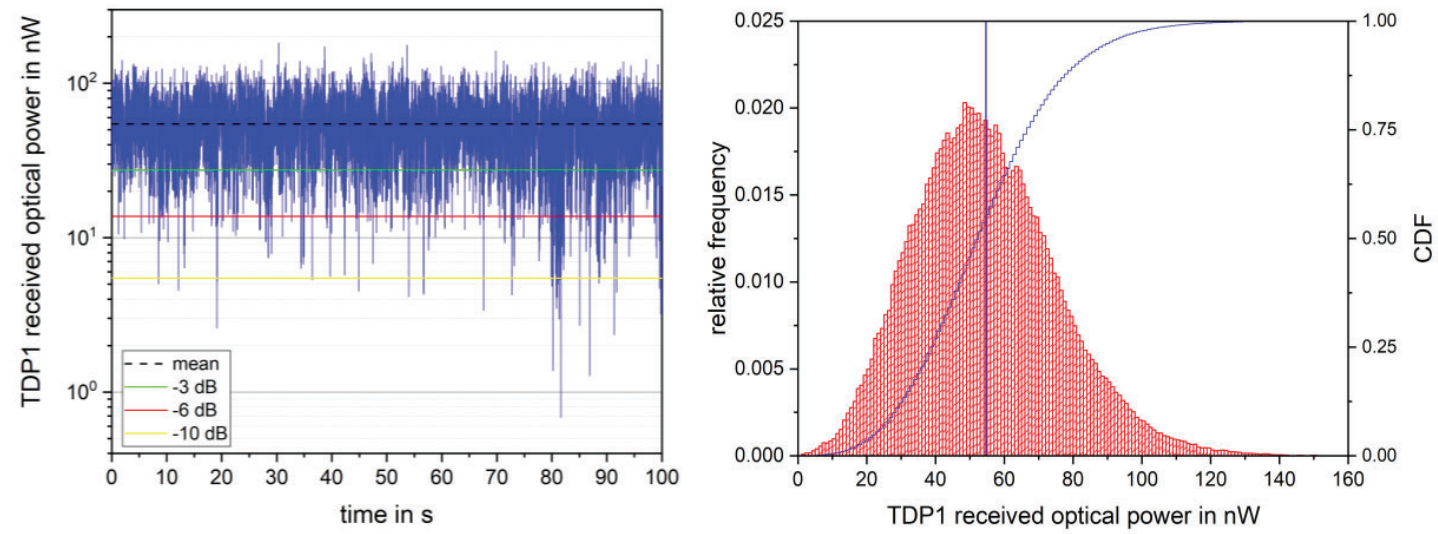

Figure 2. shows a timeline (left) and a histogram (right) of the received power at the GEO space segment TDP1-LCT. The data are recorded at: 2018-04-26 from 01-05-48 ( $=0 \mathrm{~s}$ in the plot) in a link with $\mathrm{T} 0=00: 50$. The receiver was heterodyne. The T-AOGS configuration was: CPA100 for TX and $35 \mathrm{~mm}$ sending aperture i.e. $26 \mu$ rad divergence angle. The unique CHUNK number is 2294 .

Table 3. fades statistic of a sample link, CHUNK\#2294, with mean power of $54.67 \mathrm{nW}(42.6 \mathrm{dBm})$

\begin{tabular}{|c|c|c|c|}
\hline Fade Threshold & Mean Fade Time & $\begin{array}{c}\text { Std. Dev. Fade } \\
\text { Time }\end{array}$ & $\begin{array}{c}\text { Number of } \\
\text { Fades/s }\end{array}$ \\
\hline$-3 \mathrm{~dB}$ & $6.23 \mathrm{~ms}$ & $6.28 \mathrm{~ms}$ & 12.37 \\
\hline$-6 \mathrm{~dB}$ & $4.08 \mathrm{~ms}$ & $3.78 \mathrm{~ms}$ & 1.97 \\
\hline$-10 \mathrm{~dB}$ & $3.32 \mathrm{~ms}$ & $3.53 \mathrm{~ms}$ & 0.23 \\
\hline
\end{tabular}

\subsection{Link: 2018-04-16, T0 = 21:00, T-AOGS aperture $=48$ mm, CHUNK\#2082}

The single CHUNK shown and described here shows a different, not so smooth behaviour within the optical power received at the space terminal TDP1-LCT. This time the T-AOGS performed the link using the $48 \mathrm{~mm}$ transmitter aperture. The link itself started at 21:00:00 on 2018-04-16 and $t=0$ in figure 3 represents: 21:05:59. This single CHUNK has a mean optical power received within the $135 \mathrm{~mm}$ aperture of the LCT in space of $50.86 \mathrm{nW}(-42.9 \mathrm{dBm}$, i.e. $0.36 \mathrm{nW} / \mathrm{cm} 2)$.

In the zoom (Figure 3, right) it can be seen that the mean power drifts and also varies abruptly about $\sim 3 \mathrm{~dB}$ several times for about $0.2 \mathrm{~s}$. This type of power variations is unexpected and is currently under investigation. Whether it is correlated with T-AOGS pointing (not actively changed here) or weather (which was changing that night) cannot be distinguished yet. Nevertheless: such data are also included within the remaining set of 'good data' and part of the big data analysis.

In order to measure fades independent of low frequency power variations (e.g. pointing drift) within a CHUNK, the $3 \mathrm{~dB},-6 \mathrm{~dB},-10 \mathrm{~dB}$ thresholds are not static, but relative to a $3 \mathrm{~s}$ averaged mean power. The behaviour of the local mean power in $3 \mathrm{~s}$ and the corresponding $-3 \mathrm{~dB},-6 \mathrm{~dB}$ and $-10 \mathrm{~dB}$ thresholds are also given in Figure 3. (this way of analysis is used for all CHUNKs). It can be seen (Figure 3, right) that the local mean power in $3 \mathrm{~s}$ is almost not affected by the $3 \mathrm{~dB}$ drops of $0.2 \mathrm{~s}-$ thus this type of intensity drops will increase the fade statistics.

The fades statistic as evaluated with the ATLANTIS tool of this single CHUNK is shown in Table 4. 

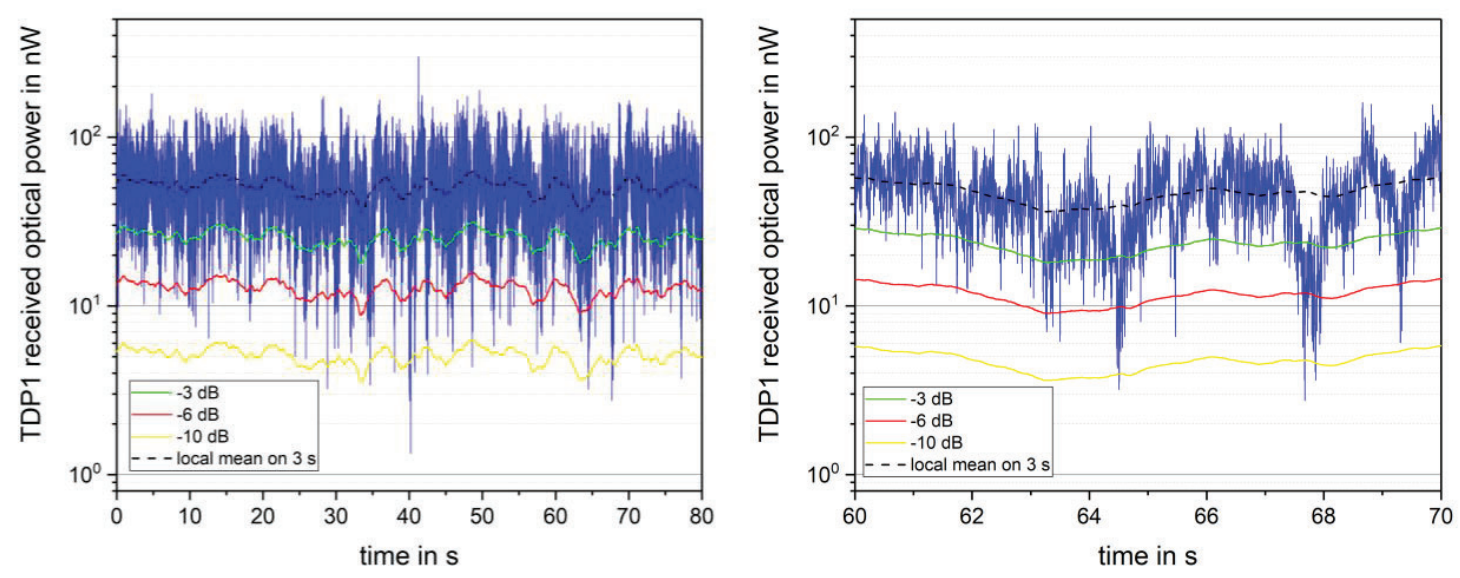

Figure 3. Timeline (left) and a zoom (right) of data recorded at 2018-04-16 from 21-05-56 with T0 = 21:00 (CHUNK \#2082). The TDP1 tracking detector is in homodyne. The T-AOGS configuration is: TX with CPA100 and aperture $48 \mathrm{~mm}$, i.e. divergence angle of $19 \mu \mathrm{rad}$ (including $20 \%$ broadening due to truncation).

Table 4. fades statistic of a sample link, CHUNK\#2028 with mean power $50.86 \mathrm{nW}$

\begin{tabular}{|c|l|l|l|}
\hline Fade Threshold & Mean Fade Time & $\begin{array}{c}\text { Std. Dev. Fade } \\
\text { Time }\end{array}$ & $\begin{array}{c}\text { Number of } \\
\text { Fades/s }\end{array}$ \\
\hline$-3 \mathrm{~dB}$ & $6.11 \mathrm{~ms}$ & $8.49 \mathrm{~ms}$ & 17.86 \\
\hline$-6 \mathrm{~dB}$ & $4.43 \mathrm{~ms}$ & $4.42 \mathrm{~ms}$ & 3.39 \\
\hline$-10 \mathrm{~dB}$ & $2.84 \mathrm{~ms}$ & $1.99 \mathrm{~ms}$ & 0.25 \\
\hline
\end{tabular}

Comparing the fades statistics of the two CHUNKs (Table 3 and Table 4), which is not only a comparison of a link performed within $35 \mathrm{~mm}$ or $48 \mathrm{~mm}$ T-AOGS aperture but also between a 'good behaviour' and a 'power variation in the range of $3 \mathrm{~dB}$, potentially due to changing attenuation within optical channel', it can be seen that the amount of $-3 \mathrm{~dB}$ fades are increased by $40 \%$, the amount of $-6 \mathrm{~dB}$ fades are increased by even $70 \%$, while the amount of $10 \mathrm{~dB}$ fades is not affected.

\section{RESULTS OF "BIG DATA" ANALYSIS}

In the following sections, results of the 'big data' analysis are shown for different questions. What is the influence of the ground segment TX beam divergence on the up-link budget: regarding mean power (section 5.1) or regarding dynamics (fade statistics in section 5.2). How does the atmosphere change over the day, here represented by Fried-Parameter $r_{0}$ as measured at the T-AOGS (section 5.3). A short insertion on the way the $r_{0}$ is measured and a possible alternative is given (section 5.4). Within section 5.5 the correlation between the $r_{0}$ measured in the down-link and the scintillation seen at the GEO orbit space segment is shown.

\subsection{Mean power at TDP1 depending on TX beam of T-AOGS}

The optical power seen at the space segment depends on the ground segment transmit (TX) beam divergence, it's pointing accuracy and the transmission of the optical channel. When analysing only mean power values, the dynamic (fades, surges) is averaged. Only beam-shape, pointing and the pure attenuation of the optical channel remain. Figure 4 
shows histograms of the mean power derived from each CHUNK (see also Figure 2, right), separated by the different TAOGS TX apertures and thus beam divergences. Without atmosphere, pointing error, and aging of the T-AOGS, each CHUNK would have the same mean power. The broadening of the distribution is caused by a slight ageing of the TAOGS transmit path, the different atmospheric transmission, and, most significantly, T-AOGS pointing error. Due to the way the T-AOGS is built (ref. $2,3,4$ ) the pointing error can easily have a semi-constant value of up to $10 \mu \mathrm{rad}$. This explains the slope to less power, as seen for all histograms. Also, the link was used for other purposes and for some of the datasets the main goal was not to achieve an optimal link budget.

The CHUNK mean power distribution for the $95 \mathrm{~mm}$ T-AOGS TX aperture (Figure 4, blue) shows, besides a pure statistic due to few links, that the broadening due to miss-pointing is huge.

In Table 5. the mean power seen in Figure 4 is given and the relation between each other, here relative to $35 \mathrm{~mm} \mathrm{~T}$ AOGS TX aperture is derived. It is compared with the relation of the different antenna gains.

The change in mean power fits almost to the theoretical factor. Additionally, one can see that the seen gain due to larger sending aperture, i.e. smaller beam divergence, is slightly less that calculated. This fits well to the influence of TX/RX misalignment and thus pointing errors, having a stronger influence for narrower beams.

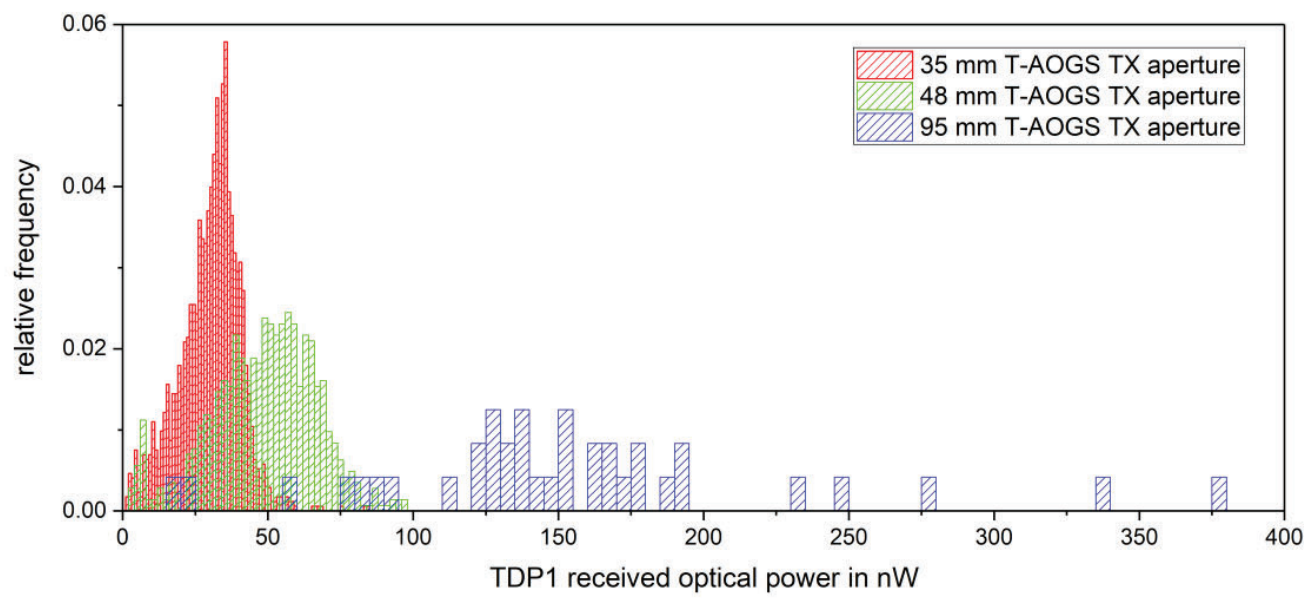

Figure 4. Distribution of the mean optical power at the space segment TDP1 derived of each CHUNK, separated by the used transmit aperture of the ground segment T-AOGS. The broadening shows the variation in the attenuation of the optical channel and the T-AOGS miss-pointing for the different CHUNKs.

Table 5. Comparison of mean power seen at the TDP1-LCT for different T-AOGS TX apertures, the relative increase due to larger sending apertures, i.e. smaller beam divergence, and the comparison with the potential gain based on calculated antenna gain.

\begin{tabular}{|c|c|c|c|c|}
\hline $\begin{array}{c}\text { T-AOGS TX } \\
\text { aperture } \\
\mathbf{2}^{*} \boldsymbol{w}_{\boldsymbol{0}}^{*} \mathbf{1 . 1 2}\end{array}$ & $\begin{array}{c}\text { Mean power } \\
\text { see Fig. } \mathbf{4}\end{array}$ & $\begin{array}{c}\text { rel. to 35mm } \\
\text { Factor (dB) }\end{array}$ & $\begin{array}{c}\text { T-AOGS } \\
\text { antenna } \\
\text { gain, } \\
\text { calculated }\end{array}$ & $\begin{array}{c}\text { 'gain' } \\
\text { relative to } \\
\mathbf{3 5 m m}\end{array}$ \\
\hline $35 \mathrm{~mm}$ & $30.3 \mathrm{nW}$ & & $99.3 \mathrm{~dB}$ & \\
\hline $48 \mathrm{~mm}$ & $48.6 \mathrm{nW}$ & $1.6(2.1 \mathrm{~dB})$ & $102.0 \mathrm{~dB}$ & $2.7 \mathrm{~dB}$ \\
\hline $95 \mathrm{~mm}$ & $153.4 \mathrm{nW}$ & $5.1(7.0 \mathrm{~dB})$ & $108.0 \mathrm{~dB}$ & $8.7 \mathrm{~dB}$ \\
\hline
\end{tabular}




\section{2 uplink: fade statistic, depending on TX divergence of T-AOGS}

Evaluating the full set of data, only filtered by the different T-AOGS TX divergence angles, average fade statistics have been derived. The statistics do not distinguish between day or night, or season of the year. As the distribution of data over the day and over the year is not balanced (see Figure 1), this average is also not fully balanced. In contrast to the single CHUNKs shown in section 3, or the data from one summer campaign shown in [7] the observed results or not any more single examples, but an overview based on large statistics. Only the numbers for links with the $95 \mathrm{~mm}$ T-AOGS transmitter aperture should be considered carefully: only a few links during noticeable good weather conditions were performed. The resulting fade statistics can be seen within Table 6, and cumulative density functions (CDF) are given in Figure 5.

Table 6. fades statistics: data separated by T-AOGS TX aperture, heterodyne and homodyne CHUNKs, data for $95 \mathrm{~mm}$ is not probing so many different times of the day - and might this be inaccurate.

\begin{tabular}{|c|c|c|c|c|c|c|c|c|c|}
\hline & \multicolumn{3}{|c|}{35 mm } & \multicolumn{3}{c|}{$48 \mathrm{~mm}$} & \multicolumn{3}{c|}{95 mm } \\
\hline $\begin{array}{c}\text { Fade } \\
\text { Threshold }\end{array}$ & $\begin{array}{c}\text { Mean } \\
\text { Fade } \\
\text { Dur. }\end{array}$ & $\begin{array}{c}\text { std } \\
\text { Fade } \\
\text { Dur. }\end{array}$ & $\begin{array}{c}\text { Numbe } \\
\text { r of } \\
\text { Fades/s }\end{array}$ & $\begin{array}{c}\text { Mean } \\
\text { Fade } \\
\text { Dur. }\end{array}$ & $\begin{array}{c}\text { std } \\
\text { Fade } \\
\text { Dur. }\end{array}$ & $\begin{array}{c}\text { Numbe } \\
\text { r of } \\
\text { Fades/s }\end{array}$ & $\begin{array}{c}\text { Mean } \\
\text { Fade } \\
\text { Dur. }\end{array}$ & $\begin{array}{c}\text { std } \\
\text { Fade } \\
\text { Dur. }\end{array}$ & $\begin{array}{c}\text { Numbe } \\
\text { r of } \\
\text { Fades/s }\end{array}$ \\
\hline$-3 \mathrm{~dB}$ & $5.8 \mathrm{~ms}$ & $8.0 \mathrm{~ms}$ & 25.7 & $6.1 \mathrm{~ms}$ & $9.2 \mathrm{~ms}$ & 27.1 & $8.9 \mathrm{~ms}$ & $16.4 \mathrm{~ms}$ & 18.7 \\
\hline$-6 \mathrm{~dB}$ & $4.2 \mathrm{~ms}$ & $7.0 \mathrm{~ms}$ & 7.5 & $4.6 \mathrm{~ms}$ & $7.0 \mathrm{~ms}$ & 9.1 & $7.0 \mathrm{~ms}$ & $16.8 \mathrm{~ms}$ & 7.8 \\
\hline$-10 \mathrm{~dB}$ & $3.2 \mathrm{~ms}$ & $9.1 \mathrm{~ms}$ & 2.0 & $3.6 \mathrm{~ms}$ & $6.8 \mathrm{~ms}$ & 2.8 & $6.2 \mathrm{~ms}$ & $12.5 \mathrm{~ms}$ & 3.2 \\
\hline
\end{tabular}
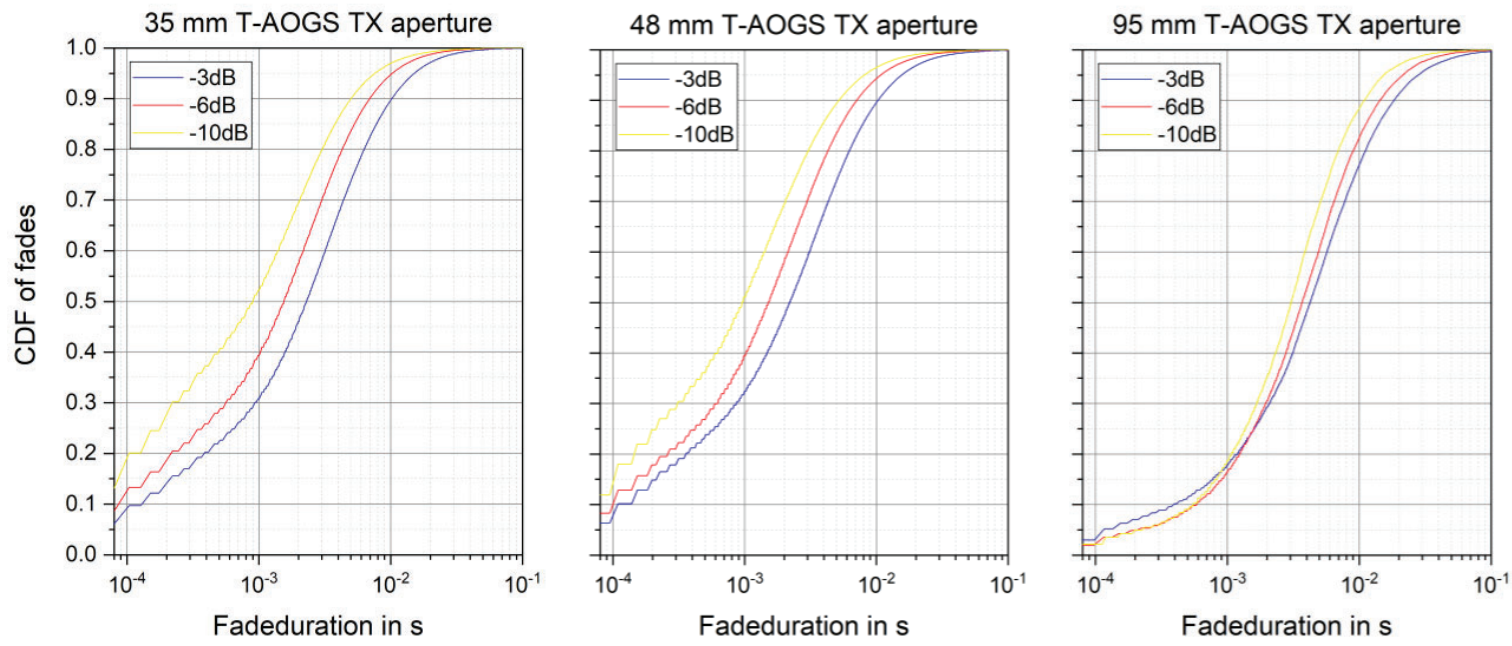

Figure 5. The cumulated density function of the numbers of fades relative to the fade duration, as seen at the space segment is shown. The CDF differs depending on the ground segments sending aperture ( $35 \mathrm{~mm}$ left, $48 \mathrm{~mm}$ middle, $95 \mathrm{~mm}$ right). the $\mathrm{CDF}$ is given for the three depth of the fades: $-3 \mathrm{~dB},-6 \mathrm{~dB}$ and $-10 \mathrm{~dB}$ relative to the actual mean power.

Comparing the fade statistics seen at TDP1 for different T-AOGS TX apertures in Table 6: The difference between $35 \mathrm{~mm}$ and $48 \mathrm{~mm}$ is only marginal .The mean fade duration increases by about $10 \%$, the number of fades per second increases by $10 \%(-3 \mathrm{~dB}), 20 \%(-6 \mathrm{~dB}), 40 \%(-10 \mathrm{~dB})$. Seeing the increase of $-10 \mathrm{~dB}$ fades of $40 \%$ when changing from $35 \mathrm{~mm}$ aperture to $48 \mathrm{~mm}$ aperture makes it obvious that, the overall link-budget, if sensitive to fades, is not necessarily improved with a larger sending aperture. The statistics on $95 \mathrm{~mm}$ sending aperture is very poor (only few links performed most of them in excellent weather condition). Nevertheless, it can be seen that, relative to $35 \mathrm{~mm}$ the mean 
fades times increases by $50 \%(-3 \mathrm{~dB}), 70 \%(-6 \mathrm{~dB}), 90 \%(-10 \mathrm{~dB})$, whereas the number of fades per second decrease for $-3 \mathrm{~dB}$ by $30 \%$, but increase for $-10 \mathrm{~dB}$ by $60 \%$. This shows that the fades get stronger with narrower beam, in-line with the expected behaviour.

Figure 5 shows the CDF of the fades relative to the fade duration, and separated to the three different T-AOGS sending apertures used. Also here it can be seen, that narrowing the beam shifts the fade duration to longer values. Less space between $-3 \mathrm{~dB},-6 \mathrm{~dB}$ and $-10 \mathrm{~dB}$ indicates that the fades get steeper: if a fade occurs it is most likely not only a $-3 \mathrm{~dB}$ fades but immediately also a $-10 \mathrm{~dB}$ fade

In order to see, which times are necessary to bridge $90 \%, 99 \%$ or even $99.9 \%$ of all fades, e.g. with an appropriate interleaver code, the relevant times are extracted from Figure 5 and given in Table 7.

Table 7. Extracted values from CDFs in Figure 5.

\begin{tabular}{|c|c|c|c|c|c|c|c|c|c|}
\hline & \multicolumn{3}{|c|}{$\begin{array}{l}90 \% \text { of fades are shorter } \\
\text { than ... in ms }\end{array}$} & \multicolumn{3}{|c|}{$\begin{array}{l}99 \% \text { of fades are shorter } \\
\text { than ... in ms }\end{array}$} & \multicolumn{3}{|c|}{$\begin{array}{c}99.9 \% \text { of fades are } \\
\text { shorter than ... in ms }\end{array}$} \\
\hline & $-3 d B$ & $-6 \mathrm{~dB}$ & $-10 \mathrm{~dB}$ & $-3 d B$ & $-6 \mathrm{~dB}$ & $-10 \mathrm{~dB}$ & $-3 \mathrm{~dB}$ & $-6 \mathrm{~dB}$ & $-10 \mathrm{~dB}$ \\
\hline $35 \mathrm{~mm}$ & 5.0 & 6.9 & 10.2 & 17.8 & 22.2 & 32.2 & 49.6 & 60.3 & 84.4 \\
\hline $48 \mathrm{~mm}$ & 5.2 & 7.1 & 10.3 & 19.5 & 24.8 & 35.6 & 61.7 & 77.6 & 108.8 \\
\hline $95 \mathrm{~mm}$ & 10.9 & 14.4 & 18.4 & 31.8 & 44.3 & 63.5 & 69.5 & 108.7 & 162.5 \\
\hline
\end{tabular}

\subsection{Fried Parameter $r_{0}$ measured at T-AOGS}

In the following section the measured Fried Parameter during operation of the T-AOGS from 2016 to 2019 at the Observatorio del Teide (Tenerife, Spain) is shown. Additionally two different calculation methods for the value are compared to each other. The Fried Parameter $r_{0}$ is a measure of the strength of atmospheric turbulence. A smaller value represents stronger turbulence than higher values. The calculation of the Fried Parameter in the T-AOGS fits the measured wave front to the theoretical Zernike mode variance spectrum. This fit can sometimes be poor due to vibrations of the T-AOGS or local dome seeing, which may not adhere to the expected statistics. This leads to an error in the calculation, which should be considered in the comparison with other calculation methods of the theoretical r0.

In contrast to the above automated extraction of data for the analysis of the following data the time spans with sufficient light and signal on the T-AOGS are chosen manually. For each of these times a mean value of the Fried Parameter is evaluated. Within Figure 6 mean values summarizing the data of a full link are shown as blue points versus the time of the day.

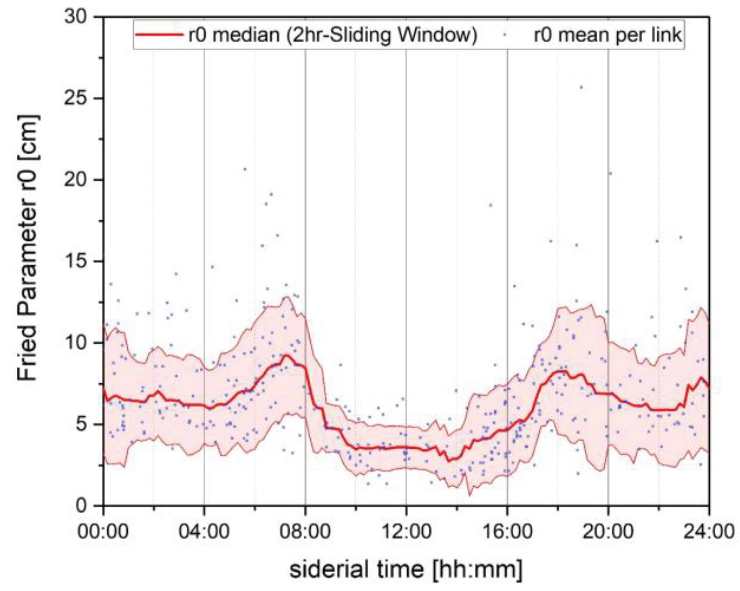

Figure 6. Fried Parameter $\mathrm{r} 0$ as measured with the T-AOGS versus time of day in sidereal time. blue: mean value of a single link. red: mean value and median within $2 \mathrm{~h}$ time-window, shifted in 10 minutes interval. Sunrise is at 6:00, sunset is at 18:00. 
There are times with more data, e.g. early afternoon, and times with less data, e.g. during lunch break but also late in the night. In order to obtain a mean behaviour over the day the single data points are subsumed in the following way: a sliding window of two hours that is shifted in ten minutes intervals is used to calculate a median and a standard deviation, given as solid red line and reddish area.

The values of r0 during the day (from 9:00 to 15:00 sidereal time) is almost constant, with a low value. During the night from 18:00 to 06:00 local sidereal time the value is also almost constant but about a factor 2 larger, i.e. less turbulence and with larger turbulence cells within the atmosphere. It is interesting to consider the time before and after sunset and sunrise, where the $\mathrm{r} 0$ rises. This has already seen and reported ${ }^{10}$, but only based on few data and the statistics becomes clearer in the presented data. In the morning r0 starts to increase shortly before sunrise and reaches a maximum (factor 2) about $1 \mathrm{~h}$ hour after sunrise. With higher altitude of the sun the $\mathrm{r} 0$ starts decreasing. $2 \mathrm{~h}$ after sunrise it is back to the 'night value' and further decreases to the 'day-value'. In the evening the main changes happen in the two hours before sun-set: $r_{0}$ increases by a factor of 2 , from the 'day level' to the 'night level' with a short peak around sun-set itself.

It is not investigated how much of the behaviour originates from the local atmosphere, i.e. turbulence coming from the ground and the T-AOGS itself due to the changing thermal conditions and how much of the behaviour originates in higher layers over the ground (often termed the "free-atmosphere").

\subsection{Comparison of Zernike based $\mathrm{r} 0$ calculation used in the T-AOGS and an alternative "Slope Covariance" based method}

The T-AOGS' internal Fried Parameter calculation is performed by fitting the measured wave-front to an atmospheric model of spatial modes. In this process, the wave-front must be "pseudo open-loop" reconstructed to determine the wave-front observed at the telescope aperture, rather than the residual wave-front as recorded by the Shack-Hartmann wave-front sensor post adaptive optics correction. The variance of the computed spatial modes is compared to a theoretical spectrum to determine the $r_{0}$ measurement. This process is subject to some errors during reconstruction of spatial modes and can be biased by vibrations of the T-AOGS and noise on the wave-front sensor.

An alternative method ${ }^{10}$ of $r_{0}$ calculation was also performed on the recorded data. Here the covariance of pseudo openloop Shack-Hartmann wave-front sensor measurements is considered and also fitted to a theoretical distribution. In this method, errors in spatial mode reconstruction are avoided and it is also possible to avoid bias due to sensor noise by neglecting the auto-covariance between sub-apertures in the fitting algorithm.

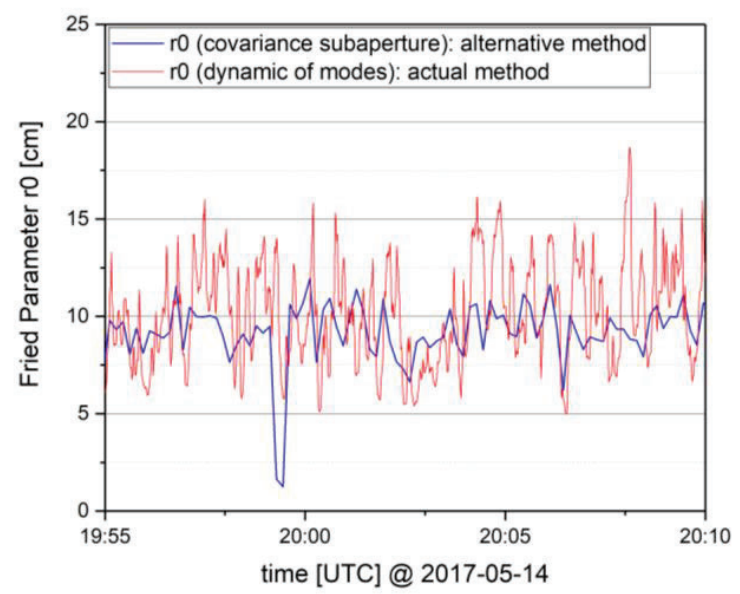

Figure 7. Comparison of two way to calculate r0: either via covariance subaperture or via dynamic modes. 
In Figure 7 it can be seen that the r0 values with the actual calculation method of the T-AOGS is slightly larger and has a higher variation than the alternative method using the covariance between sub-aperture measurements. In general the mean over several minutes has an discrepancy smaller than $1 \mathrm{~cm}$.

\subsection{Correlation between $\mathrm{r} 0$ in down-link and intensity fluctuations in the uplink}

Figure 8 shows the scintillation seen at the space segment TDP1 versus the actual Fried Parameter as measured with the T-AOGS. According to the theory there is no direct correlation. The data show a slight dependency. While a small $r_{0}$ indicates smaller turbulence cells in the atmosphere, the mean scintillation seen in space is larger. Nevertheless the scintillation spread, for a given $r_{0}$, still by a factor of 2 to 3 .

The 'dip' and 'spike' seen in the median scintillation at $\mathrm{r} 0=2 \mathrm{~cm}$ and $\mathrm{r} 0=27 \mathrm{~cm}$ are cause by small statistics and maybe indicated areas where the $r_{0}$ calculation is not reliable.

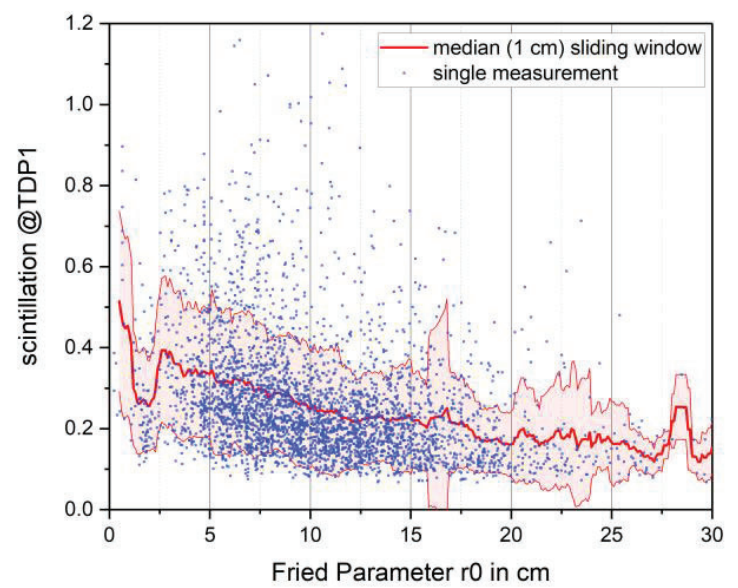

Figure 8. Scintillation seen at the space segment TDP1 versus Fried Parameter measured on ground with the T-AOGS. Blue: single measurements, performed with $35 \mathrm{~mm}$ and $48 \mathrm{~mm}$ T-AOGS TX aperture. Red: the calculated median with variance.

\section{CONCLUSION AND OUTLOOK}

Within this paper we have shown several results from analyses of the 'big data' gathered in more than 500 SGLs in the last five years. All these SGLs were executed between the T-AOGS located at Tenerife, and the TDP1-LCT on the GEO stationary spacecraft Alphasat. The measured optical channel had an elevation of $34^{\circ}+/-4^{\circ}$ (from ground). We have detailed the fades statistics, which are important for link budget considerations and designing codes for the up-link communication channel, e.g. the length of interleaver codes. The trade-off between transmitter aperture diameters were shown: smaller beam-divergence leads to higher mean power, but also stronger fades and, if the tracking and pointing system is not ideal, also larger pointing induced losses. The course of the Fried-Parameter r0 over the day is shown. To our knowledge the first time in this extent, using the same measurement method for day seeing and night seeing. The correlation between ground measured atmospheric characteristics and the dynamics seen at the space terminal has been touched upon by exploring the scintillation seen at TPD1 versus the r0 values measured on ground.

We explained the ATLANTIS tool being set up. This tool can und will be employed to investigate further questions, e.g. correlation of transmit power vs. time, but also to distinguish seasonal effects.

The T-AOGS is currently under refurbishment. One of the main changes will be that the CPA100, used for pointing, will get it's own tracking system. It is believed that this additional tracking function will lead to a better pointing and consequently less data will be affected by the T-AOGS mis-pointing. As the intensity seen at the space segment is 
always a combination of the ground segment pointing and the atmosphere, a reduction in pointing uncertainty will increase the knowledge on the effects caused by the atmosphere.

With the characterisation of the TDP1-LCT as a measurement device and the setup of the ATLANTIS tool, the GEO LCT on Alphasat is well suited and foreseen to serve for third party experiments. At the moment also a new firmware for the TDP1-LCT is prepared with several additional functions, e.g. tracking and measuring received light of slightly different wavelength than $1064 \mathrm{~nm}$.

\section{ACKNOWLEDGEMENTS}

We like to thank all people who made this project and the two systems: the TDP1-LCT and the T-AOGS happen. In a long-term project this are many - from the very beginning over the continuous financing towards the future goals. It is great that the TDP-LCT could evolve from a pure technical demonstration payload to a payload being in used for experiments and measurements of all kind and that the T-AOGS will get in it's 'second round' soon. Thanks to Synopta for the collaboration while building the $\mathrm{T}-\mathrm{AOGS}^{4}$, the ongoing refurbishment and implementation of new functionalities. Thanks to ESA and IAC in hosting the T-AOGS at Tenerife. And last but not least the DRL-RFM for the continuously support in all: from financing, to long term strategies. The activities described herein are carried out on behalf of the Space Administration of the German Aerospace Center (DLR e.V.) with funds from the German Federal Ministry of Economics and Energy with the reference number 50 YH 2001. The TDP1 Program is supported by ESA and Inmarsat.

\section{REFERENCES}

[1] D. Troendle, C. Rochow, P. Martin Pimentel, H. Zech, F. Heine, H. Kaempfner, M. Gregory, M. Motzigemba, U. Sterr, R. Meyer, M. Lutzer, S. Philipp-May "Optical LEO-GEO Data Relays: From Demonstrator to Commercial Application", AIAA Space 2014 (San Diego).

[2] Saucke, K., Seiter, C., Heine, F., Gregory, M., Tröndle, D. Fischer, E. Berkefeld, T., Feriencik, M., Feriencik, M., Richter, I., Meyer, R., “The Tesat transportable adaptive optical ground station”, Proc. SPIE 9739, FreeSpace Laser Communication and Atmospheric Propagation XXVIII, 973906 (15 March 2016)

[3] Saucke, K. et al, "The TESAT Transportable Adaptive Optical Ground Station and the operational experiences", Proceedings Volume 10562, International Conference on Space Optics — ICSO (2016)

[4] Fischer, E., Berkefeld, T., Feriencik, M., Feriencik, M., Kaltenbach, V. Soltau, D., Wandernoth, B., Czichy, R., Kunde J., Saucke, K., Heine, F., Gregory, M., Seiter, C., Kämpfner, K., "Development, Integration and Test of a Transportable Adaptive Optical Ground Station”, ICSOS 2012, New Orleans, Louisiana, U.S. (2015).

[5] Mahn, R., Saucke, K., Woicke, J,, Marynowski, T., Martin Pimentel, P., Heine, F.: „Durchführung von optischen Satellit-zu-Boden Links: Linkstatistik und Wetterauswertung aus fünf Jahren Betrieb der T-AOGS““ Deutsche Gesellschaft für Luft- und Raumfahrt - Lilienthal-Oberth e.V. (2020) https://doi.org/10.25967/530120. urn:nbn:de:101:1-2020092514244782570603

[6] Marynowski, T., Sanchez-Tercero, A., Hoepcke, N., Martín Pimentel, P., Saucke, K., Heine F., “TPD1 Laser Communication Terminal Performances and Trend Analysis: 5 Years of Service “, 25th Ka and Broadband Communications Conference, Sorento, Italy, 2019

[7] Saucke, K., Mahn, R., Martin Pimentel, P., Heine, F., Mata-Calvo, R., Surof, J., Barrios, R., Reeves, A., Bischl, H., Brandt, H. Matuz, B., "3 Years of Optical Satellite to Ground Links with the T-AOGS: Data Transmission and Characterization of Atmospheric" SPIE Vol. 11180 111801D-2, International Conference on Space Optics ICSO (2018)

[8] Martin Pimentel, P., Saucke, K., Hoepcke, N., Hasler, D., Mahn, R., Sánchez-Tercero, A., Marynowsli, T., \& Heine, F.: "LCTs 6 Years Operations: More Than 25.000 Successful Optical Links". 2019 IEEE International Conference on Space Optical Systems and Applications, ICSOS (2019)

[9] Butterley, T., Wilson, R. W., Sarazin, M.: "Determination of the profile of atmospheric optical turbulence strength from SLODAR data", Monthly Notices of the Royal Astronomical Society, Volume 369, Issue 2, June 2006, Pages 835-845, https://doi.org/10.1111/j.1365-2966.2006.10337.x”

[10] Brandt, P.N., Mauter, H.A., Smartt, R. "Day-Time Seeing Statistics at Sacramento Peak Observatory”, in "Deep Space Optical Communications", Hemmati, H., ed. (Wile, 2006), pp. 193 